\title{
A Study Determining the Efficacy of Brock String Therapy in the Treatment of Convergence Insufficiency
}

\author{
Simran Dhakal ${ }^{1 *}$, Sunaina Mary Alex ${ }^{2}$ and Sourav Karmakar ${ }^{3}$ \\ Naseema Institute of Optometry and Research, Rajiv Gandhi University of Health \\ Sciences, Bangalore, India \\ *Corresponding Author: Simran Dhakal, Naseema Institute of Optometry and \\ Research, Rajiv Gandhi University of Health Sciences, Bangalore, India.
}

Received: February 25, 2021

Published: March 10, 2021

(C) All rights are reserved by Simran Dhakal., et al.

\section{Abstract}

Aim: To determine the efficacy of brock string therapy in convergence insufficiency treatment.

Methodology: It is a prospective study where the subjects were taken from Naseema Institute of Optometry and Research. Students in the age group of 18 - 31 years meeting the criteria of inclusion and exclusion were considered. Thirty subjects with CI were studied. The following test i.e. Cover test, PBCT, NPC, NRA, positive fusional vergence, negative fusional vergence, vergence facility and accommodative facility evaluation were done. Measurements from the CI subjects were obtained before and after brock string therapy. Fifteen subjects underwent regular therapy and 15 underwent occasional therapy. Statistics were calculated using SPSS V 16 for windows. Exophoria, NPC and vergence tests were compared for before and after therapy and on between regular and occasional therapy subjects.

Results: A total of 30 students were evaluated under the age group of 18-31 years. Out of 30 subjects, 12(40\%) were males and 18 $(60 \%)$ were females. Subjects before vergence therapy were between $8 \Delta$ exophoria to $11 \Delta$ exophoria and after vergence therapy it had a range of $6 \Delta$ to $4 \Delta$. The break point for NPC improved from $7.75 \mathrm{~cm}$ to $6 \mathrm{~cm}$ in case of accommodative target and in case of non-accommodative target, it improved from $10.5 \mathrm{~cm}$ to $8 \mathrm{~cm}$. PFV break increased from $14 \Delta$ to $30 \Delta$ at near. There was significant improvement in NPC and PFV in the study.

Conclusion: Brock string therapy appears to be an easy and effective therapy for CI. Thus this study concludes that regular therapy is effective for the stability of the result.

Keywords: Convergence Insufficiency; Vision Therapy; Near Point of Convergence; Exophoria; Brock String

\section{Abbreviations}

BCVA: Best Corrected Visual Acuity; BV: Binocular Vision; CI: Convergence Insufficiency; CPM: Cycle Per Minute; CM: Centimeter; DS: Diopter Sphere; HBCVT= Home Based Computer Vision Therapy; HBPP: Home Based Pencil Pushup; NFV: Negative Fusional Vergence; NPC: Near Point of Convergence; NRA: Negative Relative Accommodation; OBVT: Office Based Vision Thera; PFV: Positive Fusional Vergence; P value: Probability Value; VT: Vision Therapy.

\section{Introduction}

Binocular single vision is the state of simultaneous vision which is achieved by the coordinated use of two eyes so that the separate and slightly different images are achieved as a single image by the process of fusion when an individual fixes his visual attention on an object of regard [1]. The main advantage of binocular vision is that it appreciates single mental image. It results in convergence (a disjugate movement), wide field of view, stereopsis. Also, the opti- 
cal defect present in one eye is made less apparent, next eye act as a safety factor against partial or complete loss of vision. Binocular vision disorders can be strabismic and non-strabismic. The most commonly encountered disorders of the binocular vision system include; non-strabismic binocular vision disorders such as convergence insufficiency, convergence excess, divergence insufficiency and divergence excess. Oculomotor dysfunctions which show inaccurate and inefficiency pursuits and saccades; while the focusing problems frequently include accommodative insufficiency, excess/ spasm, instability, infacility, and ill sustained accommodation.

Convergence insufficiency (CI) is one of the impairment of binocular vision and it is the inability to obtain or maintain adequate binocular convergence for any length of time without any undue effort and is characterized by exophoria greater at near than at distance, a receded near point of convergence, and reduced positive fusional vergence at near. Convergence is a disjugate movement in which both the eyes rotate inward so that the lines of sight intersect in front of eyes and allows bifoveal single vision to be maintained at any fixation distance. The prevalence of $\mathrm{CI}$ is reported to range between $3-5 \%$ of the population, according to the WHO (World Health Organization). Convergence Insufficiency- A Major Review by Cooper and Jamal (2012) reported variable prevalence rates as high as 33\% [2]. Some have estimated its prevalence to be as high as $8.3 \%$ in school- age children and university students $[3,4]$.

The exact etiology of convergence insufficiency is still unknown. It may be associated with a wide inter pupillary distance (IPD), inadequate functional development, overwork, worry, uncorrected high hypermetropia or myopia, extraocular muscles imbalance, etc. People may develop the symptoms like eyestrain, blurred near vision, intermittent crossed diplopia, itching soreness and burning of eyes, trouble in changing focus from distant to near object, etc.

These problems can be treated only by exercise/therapy and these therapies are known as vision therapy (VT). Vision therapy is an array of neurosensory and neuromuscular activities individually prescribed and monitored by the doctor or optometrist to develop, rehabilitate and enhance visual skills and processing. It is based on the results of a comprehensive eye examination or consultation, and takes into consideration the results of standardized tests, the needs of the patient, and the patient's signs and symptoms [5]. Different types of VT available are home based therapy, office based therapy and computer based therapy. Some of the manual vision therapies are: pencil push-ups, red filter convergence exercises, vectograms, brock string exercise, etc.

\section{Brock string}

Brock string (named after Frederick W. Brock) is an instrument which is used in vision therapy. It consists of a white string of approximately 7 to 10 feet in length with three small beads of different colors.

The Brock string is commonly employed during treatment of anomalies of binocular vision. It is used to develop skills of convergence and also to disrupt suppression of one of the eyes.

During therapy, one end of the Brock string is held on the nose tip while the other is tied to a fixed point. The three beads are placed at various distances and the patient must focus on one of the beads, while noting the visual input of each eye and sensation of convergence. Patient should focus on the bead but simultaneously he should look at the rope. The patient can use variable techniques to make easier or more difficult by bringing the beads closer/further to the nose and also by employing lenses and prisms.

Convergence insufficiency is a treatable binocular vision disorder that can be successfully managed with in-office vision therapy as well as with pencil push-ups and computer orthoptics software. It appears that patients continue to experience symptomatic relief as well as improvement in positive fusional vergence and the near point of convergence up to a year post-treatment [6].

So, in this study, brock string exercise is used as this simple device can be helpful to improve accuracy of focusing, fixation, convergence, speed of convergence and divergence, fusional reserves, visual spatial accuracy, speed of eye movements and vergences, and anticipation. It is also excellent as an anti-suppression device and requires good central and peripheral integration and freedom from distraction. Also, there are very few studies about brock string exercise.

\section{Aim of the Study}

- To determine the efficacy of brock string therapy in convergence insufficiency treatment.

\section{Objectives}

- To assess the need of vision therapy among convergence insufficiency subjects 
- To compare the efficacy of brock string exercise in regular and occasional subjects.

\section{Materials and Methods \\ Study population}

Normal subjects.

Study design

Prospective study (Cross sectional).

Sample size: 30

$\mathrm{n}=\left(\mathrm{Z}_{1-\alpha / 2}+\mathrm{Z}_{1-\beta / 2}\right)^{2} \sigma^{2} / \mathrm{d}^{2}$

$\mathrm{Z}_{1-\alpha / 2}=1.96$ ( for $95 \%$ confidence interval)

$\mathrm{Z}_{1-\beta}=0.84$ (as hypothetical testing was based on $80 \%$ power)

Standard deviation $(\sigma)=9.7$

Mean of difference $(d)=5$

$\mathrm{n}=(1.96+0.84)^{2}(9.7)^{2} / 5^{2}$

$\mathrm{n}=30$.

Study duration

October 2016- May 2017.

Sampling technique

Simple random sampling.

Data analysis

SPSS software.

\section{Inclusion criteria}

- Subject with exophoria $>6 \Delta$ at near (according to Morgan's criterion)

- NPC with non-accommodative target $>9 \mathrm{~cm}$.

\section{Exclusion criteria}

- Subjects with a primary accommodation disorder

- Subjects with any systemic or ocular disease

- Subjects who are intellectually challenged.

\section{Assessment}

For screening the vision, we used Log MAR visual acuity for both distance and near.
In binocular vision assessment, near point of convergence (NPC) was measured with accommodative target $\left(\mathrm{N}_{8}\right.$ letter size) and nonaccommodative target (red filter lens and torch light). Cover test was done at both distance and near with the help of hand held occluder and measured with prism bar if there was any deviation. Ocular motility was checked using Broad $\mathrm{H}$ test. Binocular vision sensory status was evaluated by worth 4 dot test at distance, intermediate and near and stereopsis was evaluated using random dot stereogram. Negative relative accommodation (NRA) and positive relative accommodation (PRA) was done to check the state of accommodation (directly) and also to find the vergence disorders (indirectly). Negative fusional vergence (NFV) and positive fusional vergence (PFV) was done at both distance and near to check the amplitude of divergence and convergence respectively. Accommodative facility was assessed binocularly using accommodative flipper to find the speed of relaxation and stimulation of accommodation and also to evaluate vergence anomalies. Vergence facility was done to find the speed of convergence and divergence.

\section{Methodology}

Subjects were selected from Naseema Institute of Optometry and Research of Bangalore from October 2016 to May 2017. The purpose of the study was explained and informed consent was taken from them.

All subjects had to undergo a comprehensive ocular examination including BCVA. Eyes that fulfil the inclusion criteria were selected for further assessment and 30 subjects were selected for this study. Brock string therapy was given on regular basis and occasional basis (weekly thrice; therapy every alternate days) till exophoria is $\leq 6 \Delta$ and NPC $\leq 9$ $\mathrm{cm}$. 15 subjects were included in both the groups. White string of approximately 7 feet was used with green and yellow color beads. Firstly, the patient had to focus on green bead and then on yellow bead. The beads were moved towards the patient on 4 steps and the procedure was repeated as before. Vergence tests were done in every 2 weeks. When exophoria and NPC values gets normal, therapy was discontinued. To check the consistency of therapy, binocular vision assessment was done after a month.

Below is the detail procedure which is performed during the study.

Screening

Visual acuity

Monocular and binocular visual acuity was measured and record- 
exceeded $8 \Delta \mathrm{D}$ at distance and $6 \Delta \mathrm{D}$ at near. The normal deviation of exophoria was checked at distance.

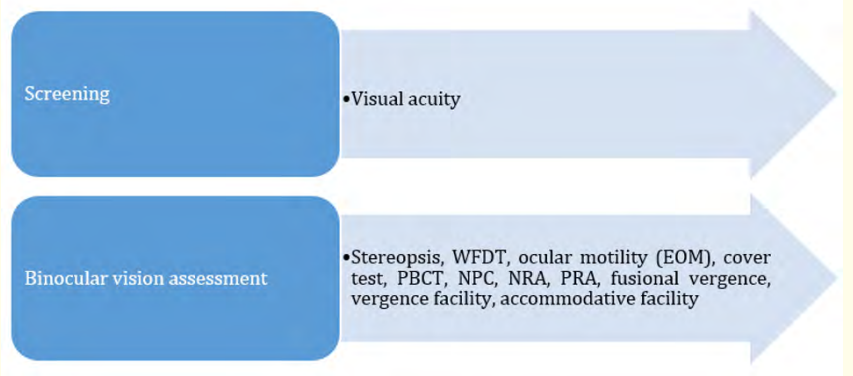

Figure a

ed with log MAR chart at distance under normal lighting condition. Near visual acuity was also measured at $30-35 \mathrm{~cm}$ with near vision card.

\section{Binocular vision examination}

- Stereopsis: Stereopsis was checked using random dot stereogram. Stereopsis equal to 40-60 sec of arc was considered as normal.

- Worth's Four Dot Test (WFDT): Binocular single vision was checked using WFDT. It was done at all distances. The subjects had to wear red green goggles and if the subject sees 5 dots, he/ she is having diplopia, if he/she sees 2 red or 3 green dots then he/she is having suppression. If he/she sees red and green dots alternatively then the subject is having alternate suppression.

- Near point of convergence: Near point of convergence was measured with an accommodative target $\left(\mathrm{N}_{8}\right.$ letter size $)$ at 40 $\mathrm{cm}$ by moving the target along the scale towards the eye. For non-accommodative target, the torch light was shone at $40 \mathrm{~cm}$ distance and slowly moved towards the eye. Convergence of less than $9 \mathrm{~cm}$ was considered normal and $>9 \mathrm{~cm}$ was considered to be defective. This test was repeated more than 3 times. Both objective (divergence of any one eye after suppression) as well as subjective (patient reports two vertical lines) findings were recorded.

- Cover test: Ocular alignment was assessed by means of cover test at $6 \mathrm{~m}$ and at $40 \mathrm{~cm}$ for distance and near respectively. No movement on cover test was referred as orthophoria. Exophoria was considered significant when outward latent deviation
- Broad H test: Ocular motility was assessed by means of broad H test.

- Relative amplitude accommodation: This test is a measure of total amount of accommodation when the eye can exert while the convergence of the eyes is fixed. It can be positive, known as positive relative accommodation (PRA) and negative, known as negative relative accommodation (NRA). The N8 target size was kept at $40 \mathrm{~cm}$. The subjects were asked to keep the near target clear and single while examiner increases the lens in steps of $0.25 \mathrm{DS}$ binocularly till the patient reports first sustained blur or double. To measure NRA, convex lenses were used and to measure PRA concave lenses were used. If the value is less than +2.50 D (low NRA), convergence insufficiency (CI) was considered significant.

- Fusional vergence: This test is done to check amplitude of convergence and divergence. Convergence amplitude (PFV) was tested using base out (BO) prism and divergence amplitude (NFV) using base in (BI) prism. Fusional vergence disorder is considered significant if PFV was less than $18 \Delta$ for both distance and near and if NFV was less than $7 \Delta$ and $14 \Delta$ for distance and near respectively. It was done with the help of prism bar and accommodative target (distance and near). Prism bar was placed in front of one eye and power was gradually increased and patient was asked to report when the target becomes blur (Blur), when it becomes double (Break), and then power of prism was decreased till the patient reports the target single again (Recovery). All three values were noted down for distance and near.

- Vergence facility: Convergence and divergence speed has been assessed by means of vergence flipper with $\mathrm{BO}$ and $\mathrm{BI}$ prisms. It was considered as abnormality if the value was less than 12 cpm. In current study vergence facility was measured using 3 base in and 12 base out prism. The test was performed for 1 minute and cycle per minute was calculated by number of flips.

- Accommodative facility: This is to evaluate the dynamics of accommodative responses under monocular and binocular condition. In our study, it was done with the help of \pm 2 DS flipper using near accommodative rock card as a target. Us- 
ing this as target, the examiner asked the patient to read the word after each flip of the lens. The test was performed for 1 minute and cycle per minute was calculated by number of flips $(1 \mathrm{cpm}=1$ plus side clearance and 1 minus side clearance). This test was done only binocularly and convergence insufficiency is considered significant if it is less than $12 \mathrm{cpm}$.

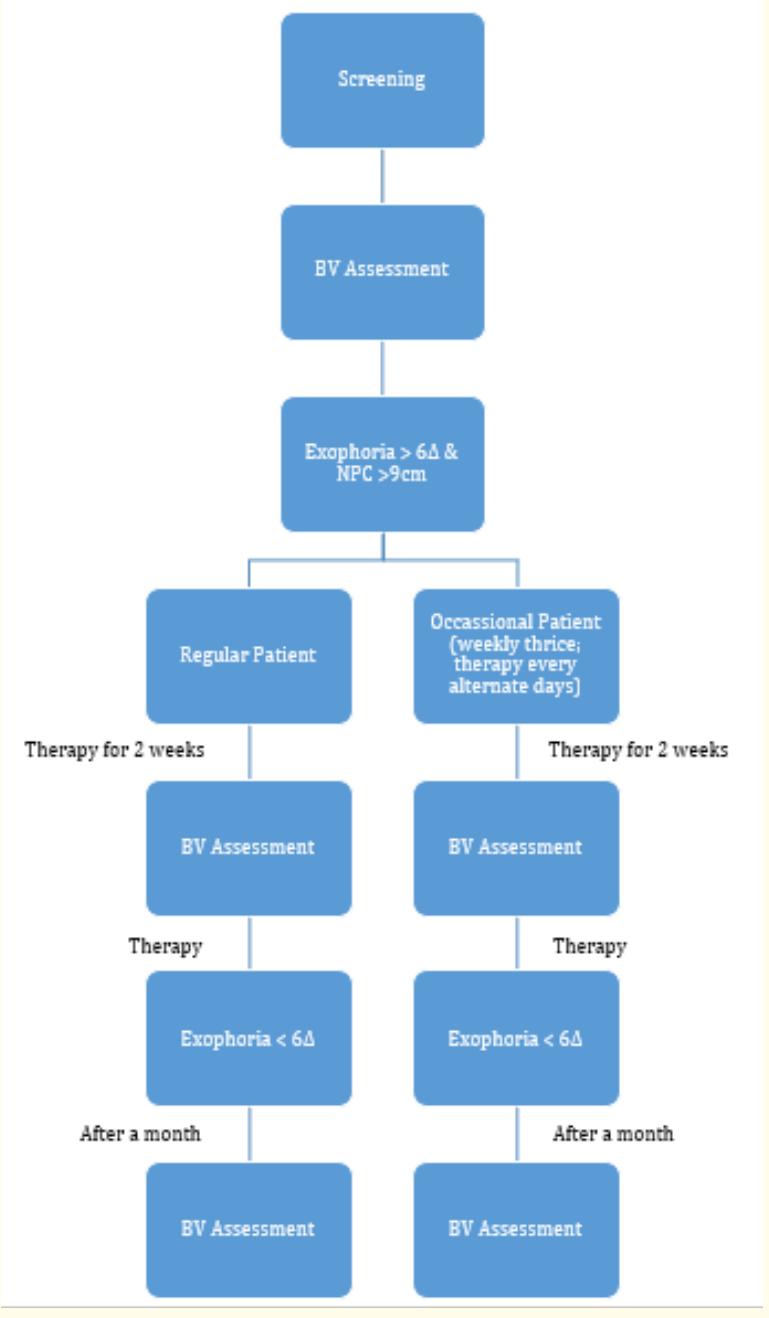

Figure b

\section{Results and Discussion}

There were two primary types of measurements investigated within this study. The first was near exophoria and the second was NPC. Along with this, the direct and indirect vergence measurements were also evaluated.
The subject's data were stratified into two groups: CI subjects before and after vergence therapy and the same subjects after the interval of 1 month of therapy. Descriptive analysis was conducted to study the following: (1) the comparison of variables between before and after therapy and (2) the comparison between regular and occasional subjects; Significance was defined as a $\mathrm{p}<0.05$. Statistics were calculated using SPSS V 16 for windows. Comparison of outcome variables between before and after treatment were compared by Whilcoxon-signed rank test and Mann-Whitney U test used to compare the difference in change before and after values between occasional and regular patients.

30 subjects were selected for the study out of which 18 were female and 12 were male. The lowest age taken was 18 years and the highest age taken was 33 years. The average stereopsis with one standard deviation was $44.33 \mathrm{sec}$ of $\operatorname{arc} \pm 5.040 \mathrm{sec}$ of arc.

\section{Statistical analysis}

Demographical details of the study subjects

\begin{tabular}{|l|c|c|}
\hline Age groups & Frequency & Percent \\
\hline$<25$ & 27 & 90.0 \\
\hline $26-30$ & 1 & 3.3 \\
\hline $31-35$ & 2 & 6.7 \\
\hline Total & 30 & 100.0 \\
\hline
\end{tabular}

Table 1: Age distribution of the study subjects.

The mean age of the study population was $21.8 \pm 3.2$ years. $90 \%$ of the subjects belong to the age group of 19-25 years.

\begin{tabular}{|l|c|c|}
\hline Gender & Frequency & Percent \\
\hline Females & 18 & 60.0 \\
\hline Males & 12 & 40.0 \\
\hline Total & 30 & 100.0 \\
\hline
\end{tabular}

Table 2: Gender distribution of the study subjects. $60 \%$ of the subjects were females and $40 \%$ were males.

Comparison of various study outcomes before and after the brock string exercise

There was a decrease in PBCT near, improvement in NPC with both accommodative and with non-accommodative target after 
98

\begin{tabular}{|c|c|c|c|c|c|}
\hline \multirow{2}{*}{\multicolumn{2}{|c|}{ Variables }} & \multicolumn{2}{|c|}{ 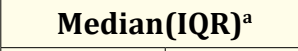 } & \multirow{2}{*}{$\mathbf{Z}^{\mathbf{b}}$} & \multirow{2}{*}{$P$ value } \\
\hline & & Before & After & & \\
\hline \multicolumn{2}{|c|}{ PBCT near } & 9 & 5 & -4.8 & $<0.001^{*}$ \\
\hline \multirow{2}{*}{$\begin{array}{l}\mathrm{NPC} \\
(\mathrm{cm})\end{array}$} & $\begin{array}{l}\text { With acc } \\
\text { target }\end{array}$ & $\begin{array}{c}7.75 \\
(0-10)\end{array}$ & $\begin{array}{c}6 \\
(0-8.3)\end{array}$ & -3.7 & $<0.001^{*}$ \\
\hline & $\begin{array}{l}\text { With non } \\
\text { acc target }\end{array}$ & $\begin{array}{c}10.5 \\
(9.8-12.3)\end{array}$ & $\begin{array}{c}8 \\
(7-9)\end{array}$ & -4.8 & $<0.001^{*}$ \\
\hline \multicolumn{2}{|c|}{ NRA(DS) } & $\begin{array}{c}2 \\
(1.75-2.25)\end{array}$ & $\begin{array}{c}2.5 \\
(2.5-2.5)\end{array}$ & -4.8 & $<0.001^{*}$ \\
\hline
\end{tabular}

Table 3: Comparison of PBCT, NPC and NRA before and after the brock string exercise.

a. Inter quartile range; b.Wilcoxon Signed Ranks Test;

*statistically significant at $\alpha=5 \%$

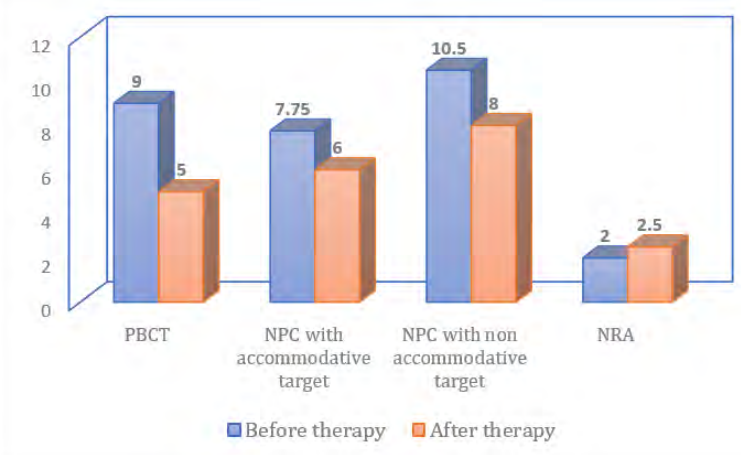

Figure 1: Comparison of PBCT, NPC and NRA before and after the brock string exercise.

brock string exercise compared to those before brock string exercise. NRA increased after intervention compared to that before. All of these results were statistically significant $(\mathrm{P}<0.05)$.

The was an improvement in all the variables, except PFV and NFV near blur vision after brock string exercise compared to those before brock string exercise and all of these results were statistically significant $(\mathrm{P}<0.05)$.

\begin{tabular}{|c|c|c|c|c|c|c|}
\hline \multirow{2}{*}{\multicolumn{3}{|c|}{ Variables }} & \multicolumn{2}{|c|}{ Median(IQR) $^{a}$} & \multirow{3}{*}{$\begin{array}{c}\mathbf{Z}^{\mathbf{b}} \\
-3.9\end{array}$} & \multirow{3}{*}{$\begin{array}{l}\text { P value } \\
<0.001^{*}\end{array}$} \\
\hline & & & \multirow{2}{*}{$\begin{array}{c}\text { Before } \\
0 \\
(0-0)\end{array}$} & \multirow{2}{*}{$\begin{array}{c}\text { After } \\
12 \\
(0-16)\end{array}$} & & \\
\hline \multirow{6}{*}{ NFV } & \multirow{3}{*}{ Distance } & Blur & & & & \\
\hline & & Break & $\begin{array}{c}10 \\
(8-12)\end{array}$ & $\begin{array}{c}18 \\
(14-18) \\
\end{array}$ & -4.8 & $<0.001^{*}$ \\
\hline & & Recovery & $\begin{array}{c}8 \\
(6-10) \\
\end{array}$ & $\begin{array}{c}4 \\
(10-16) \\
\end{array}$ & -4.8 & $<0.001^{*}$ \\
\hline & \multirow{3}{*}{ Near } & Blur & 0 & $\begin{array}{c}0 \\
(0-15 \\
\end{array}$ & -1.7 & 0.07 \\
\hline & & Break & $\begin{array}{c}12 \\
(10-16)\end{array}$ & $\begin{array}{c}19 \\
(18-25) \\
\end{array}$ & -4.6 & $<0.001^{*}$ \\
\hline & & Recovery & $\begin{array}{c}10 \\
(8-12.5)\end{array}$ & $\begin{array}{c}19 \\
(18-25) \\
\end{array}$ & -4.8 & $>0.001^{*}$ \\
\hline \multirow{6}{*}{ PFV } & \multirow{3}{*}{ distance } & Blur & 0 & $\begin{array}{c}0 \\
(0-18) \\
\end{array}$ & -2.9 & $0.003^{*}$ \\
\hline & & Break & $\begin{array}{c}12 \\
(11.5-16) \\
\end{array}$ & $\begin{array}{c}20 \\
(20-25) \\
\end{array}$ & -2.1 & $0.03^{*}$ \\
\hline & & Recovery & $\begin{array}{c}8 \\
(6-10) \\
\end{array}$ & $\begin{array}{c}18 \\
(16-20) \\
\end{array}$ & -4.8 & $<0.001^{*}$ \\
\hline & \multirow{3}{*}{ Near } & Blur & $\begin{array}{c}0 \\
(0-10.5) \\
\end{array}$ & $\begin{array}{c}0 \\
(0-0) \\
\end{array}$ & -1.4 & 0.15 \\
\hline & & Break & $\begin{array}{c}14 \\
(12-16)\end{array}$ & $\begin{array}{c}30 \\
(25-35) \\
\end{array}$ & -4.9 & $<0.001^{*}$ \\
\hline & & Recovery & $\begin{array}{c}12 \\
(10-14)\end{array}$ & $\begin{array}{c}25 \\
(20-30)\end{array}$ & -4.7 & $<0.001^{*}$ \\
\hline
\end{tabular}

Table 4: Comparison of variables related to Vergence amplitude before and after the brock string exercise.

a. Inter quartile range; b. Wilcoxon Signed Ranks Test;

*statistically significant at $\alpha=5 \%$

\begin{tabular}{|l|c|c|c|c|}
\hline \multirow{2}{*}{ Variables } & \multicolumn{2}{|c|}{ Median(IQR) $^{\mathbf{a}}$} & \multirow{2}{*}{$\mathbf{Z}^{\mathbf{b}}$} & \multirow{2}{*}{ P value } \\
\cline { 2 - 3 } & Before & After & & \\
\hline $\begin{array}{l}\text { Vergence } \\
\text { facility(cpm) }\end{array}$ & $\begin{array}{c}9.5 \\
(8-10)\end{array}$ & $\begin{array}{c}14 \\
(13-15)\end{array}$ & -4.8 & $<0.001^{*}$ \\
\hline $\begin{array}{l}\text { Accommodative } \\
\text { facility(com) }\end{array}$ & $\begin{array}{c}9.5 \\
(7.3-10)\end{array}$ & $\begin{array}{c}12 \\
(10.5-13)\end{array}$ & -4.6 & $<0.001^{*}$ \\
\hline
\end{tabular}

Table 5: Comparison of Vergence facility and Accommodative facility before and after the brock string exercise.

a. Inter quartile range; b. Wilcoxon Signed Ranks Test;

*statistically significant at $\alpha=5 \%$ 
Vergence and accommodative facilities were significantly $(\mathrm{P}<$ 0.05 ) increased after brock string exercise compared to those before brock string exercise.

Comparison of the efficacy of brock string exercise in regular and occasional subjects

\begin{tabular}{|c|c|c|c|c|c|}
\hline \multirow{2}{*}{\multicolumn{2}{|c|}{ Variables }} & \multicolumn{2}{|c|}{$\begin{array}{l}\text { Difference between before } \\
\text { and after treatment } \\
\text { (After -Before) } \\
\text { Median(IQR) }^{\mathrm{a}}\end{array}$} & \multirow[t]{2}{*}{$\mathbf{Z}^{\mathbf{b}}$} & \multirow[t]{2}{*}{$P$ value } \\
\hline & & Occasional & Regular & & \\
\hline \multicolumn{2}{|c|}{ PBCT near } & $\begin{array}{c}-3 \\
(-5 \text { to }-2)\end{array}$ & $\begin{array}{c}-5 \\
(-7 \text { to }-4)\end{array}$ & -2.44 & $0.01 *$ \\
\hline \multirow{2}{*}{$\begin{array}{l}\mathrm{NPC} \\
\mathrm{cm}\end{array}$} & $\begin{array}{c}\text { With acc } \\
\text { target }\end{array}$ & $\begin{array}{c}0 \\
(-1 \text { to } 0)\end{array}$ & $\begin{array}{c}-2 \\
(-2.5 \text { to } 0) \\
\end{array}$ & -2.49 & $0.01^{*}$ \\
\hline & $\begin{array}{l}\text { With non } \\
\text { acc target }\end{array}$ & $\begin{array}{c}-2 \\
(-3 \text { to }-1)\end{array}$ & $\begin{array}{c}-4 \\
(-5 \text { to }-2)\end{array}$ & -3.4 & $<0.001^{*}$ \\
\hline \multicolumn{2}{|c|}{ NRA(DS) } & $\begin{array}{c}0.5 \\
(0.25-0.5)\end{array}$ & $\begin{array}{c}0.5 \\
(0.5 \text { to } 0.75)\end{array}$ & -1.74 & 0.08 \\
\hline
\end{tabular}

Table 6: Comparison of PBCT, NPC and NRA change happened before and after a month of the brock string exercise between regular and occasional subjects.

a. Inter quartile range; b. Mann-Whitney U Test;

*statistically significant at $\alpha=5 \%$.

The above table shows that the difference in PBCT near, NPC with accommodative and non-accommodative target and NRA between before and after treatment were significantly $(\mathrm{p}<0.05)$ observed more among patients who underwent regular brock string therapy than that among patients underwent occasional therapy.

The above table shows that the difference in NFV distance blur, break and PFV distance break, near and break recovery values between before and after treatment were significantly $(\mathrm{p}<0.05)$ observed more among patients who underwent regular brock string therapy than that among patients underwent occasional therapy.

The difference in accommodative facility between before and after treatment were significantly $(\mathrm{p}<0.05)$ more among patients who underwent regular brock string exercise than that among patients underwent occasional exercise.

\begin{tabular}{|c|c|c|c|c|c|c|}
\hline \multirow{2}{*}{\multicolumn{3}{|c|}{ Variables }} & \multicolumn{2}{|c|}{$\begin{array}{c}\text { Difference between } \\
\text { before and after } \\
\text { treatment } \\
\text { (After -Before) } \\
\text { Median(IQR) }^{\mathrm{a}}\end{array}$} & \multirow{3}{*}{\begin{tabular}{|c}
$\mathbf{Z}^{\mathbf{b}}$ \\
-2.13
\end{tabular}} & \multirow{3}{*}{\begin{tabular}{|c} 
P value \\
$0.03^{*}$
\end{tabular}} \\
\hline & & & \multirow{2}{*}{\begin{tabular}{|c|} 
Occasional \\
6 \\
$(0-12)$
\end{tabular}} & \multirow{2}{*}{$\begin{array}{c}\text { Regular } \\
14 \\
(4-16)\end{array}$} & & \\
\hline \multirow{6}{*}{ NFV } & \multirow{3}{*}{ Distance } & Blur & & & & \\
\hline & & Break & $\begin{array}{c}6 \\
(4-8)\end{array}$ & $\begin{array}{c}8 \\
(6-10)\end{array}$ & -1.92 & $0.05^{*}$ \\
\hline & & Recovery & $\begin{array}{c}6 \\
(4-6) \\
\end{array}$ & $\begin{array}{c}6 \\
(4-8) \\
\end{array}$ & -1.45 & 0.15 \\
\hline & \multirow{3}{*}{ Near } & Blur & 0 & $\begin{array}{c}0 \\
(0-16)\end{array}$ & -1.39 & 0.17 \\
\hline & & Break & $\begin{array}{c}8 \\
(4-10)\end{array}$ & $\begin{array}{c}8 \\
(6-10)\end{array}$ & -0.69 & 0.49 \\
\hline & & Recovery & $\begin{array}{c}6 \\
(2-9)\end{array}$ & $\begin{array}{c}6 \\
(6-10)\end{array}$ & -0.92 & 0.36 \\
\hline \multirow{6}{*}{ PFV } & \multirow{3}{*}{ Distance } & Blur & $\begin{array}{c}0 \\
(0-18)\end{array}$ & $\begin{array}{c}0 \\
(0-16)\end{array}$ & -0.39 & 0.70 \\
\hline & & Break & $\begin{array}{c}8 \\
(6-13) \\
\end{array}$ & $\begin{array}{c}13 \\
(8-16)\end{array}$ & -2.15 & $0.03^{*}$ \\
\hline & & Recovery & $\begin{array}{c}6 \\
(6-10)\end{array}$ & $\begin{array}{c}10 \\
(6-13)\end{array}$ & -1.83 & 0.07 \\
\hline & \multirow{3}{*}{ Near } & Blur & $\begin{array}{c}0 \\
(-12-0) \\
\end{array}$ & $\begin{array}{c}0 \\
(0-0) \\
\end{array}$ & -0.84 & 0.40 \\
\hline & & Break & $\begin{array}{c}11 \\
(9-14)\end{array}$ & $\begin{array}{c}19 \\
(16-25)\end{array}$ & -3.66 & $<0.001^{*}$ \\
\hline & & Recovery & $\begin{array}{c}10 \\
(7-11)\end{array}$ & $\begin{array}{c}18 \\
(13-24)\end{array}$ & -3.77 & $<0.001 *$ \\
\hline
\end{tabular}

Table 7: Comparison of change happened in variables related to Vergence amplitude before and after a month of the brock string therapy between regular and occasional subjects. a. Inter quartile range; b. Mann-Whitney U Test. *statistically significant at $\alpha=5 \%$.

Summary

- Improvement in NPC and vergence tests has shown significance in both groups with a p value $<0.05$. 


\begin{tabular}{|c|c|c|c|c|}
\hline \multirow[t]{2}{*}{ Variables } & \multicolumn{2}{|c|}{$\begin{array}{c}\text { Difference between } \\
\text { before and after } \\
\text { treatment } \\
\text { (After -Before) }^{\text {Median(IQR) }} \\
\end{array}$} & \multirow[t]{2}{*}{$\mathbf{Z}^{\mathbf{b}}$} & \multirow[t]{2}{*}{$\begin{array}{c}P \\
\text { value }\end{array}$} \\
\hline & Occasional & Regular & & \\
\hline $\begin{array}{l}\text { Vergence } \\
\text { facility (cpm) }\end{array}$ & $\begin{array}{c}4 \\
(3-5)\end{array}$ & $\begin{array}{c}5 \\
(3-7)\end{array}$ & -1.53 & 0.13 \\
\hline $\begin{array}{l}\text { Accommodative } \\
\text { facility (cpm) }\end{array}$ & $\begin{array}{c}3 \\
(1-4)\end{array}$ & $\begin{array}{c}4 \\
(3-5)\end{array}$ & -2.17 & $0.03^{*}$ \\
\hline
\end{tabular}

Table 8: Comparison of change happened in vergence facility and Accommodative facility before and after a month of the brock string exercise between regular and occasional subjects. a. Inter quartile range; b. Mann-Whitney U Test; *statistically significant at $\alpha=5 \%$.

- Among the types of therapy in our study, regular therapy is found to be clinically and statistically significant.

\section{Discussion}

This study was designed to determine the efficacy of vision therapy particularly brock string exercise in the treatment of convergence insufficiency. Office-based vision therapy is expensive for underprivileged people, needs specialized equipment necessary for a full course and also may not be locally available. So, in these cases, a viable alternative would be to prescribe home-based VT (HBVT). There are very few studies in brock string therapy which is also a HBVT, this study aims in determining the efficacy of brock string therapy. The results of this study demonstrate that brock string therapy can result in both clinically and statistically significant changes in NPC with corresponding improvement in vergence tests.

One of the significant result of this study was that the near dissociated phoria (exophoria) was eliminated after vergence therapy. Subjects before brock string therapy were between $8 \Delta$ exophoria to $11 \Delta$ exophoria where the median was $9 \Delta$. The exophoria after brock string therapy for the CI subjects had a range of $6 \Delta$ to $4 \Delta$ where the median was $5 \Delta$. A similar type result was found in a study done among four CI subjects who before vergence therapy were between $8 \Delta$ exophoria to $10 \Delta$ exophoria where the average with one stan- dard deviation was $9.5 \Delta$ exophoria $\pm 0.1 \Delta$. After vergence therapy, it had a range of $6 \Delta$ to $2 \Delta$ exophoria where the average and one standard deviation was $3.5 \Delta$ exophoria $\pm 1.9 \Delta$. The laboratory and home therapy consisted of step and ramp stimuli [7].

In a study by Aziz S, there was an improvement in NPC from $16.6 \mathrm{~cm}$ to $8.4 \mathrm{~cm}$ after orthoptics therapy which was performed for 8.2 months. This study concluded that orthoptic exercises are an effective means of reducing symptoms in patient with $\mathrm{CI}$ along with improvement in NPC [8]. Schieman., et al. found that the NPC break improved in the vision therapy/orthoptics group decreasing from $12.8 \mathrm{~cm}$ to $5.3 \mathrm{~cm}$. The PFV break at near increased significantly in the vision therapy/orthoptics group from $11.3 \Delta \pm 4.3$ to $29.7 \Delta$ \pm 10.8 ( $\mathrm{p}=0.001$ ) [9]. In a comparison between $\mathrm{BI}$ prism reading glass and placebo reading glass, there was an improvement in NPC from $17.95 \mathrm{~cm}$ to $13.81 \mathrm{~cm}$ in case BI prism reading glass group and from $15.87 \mathrm{~cm}$ to $14.54 \mathrm{~cm}$ in case of placebo reading glass group. PFV at near increased from $10.55 \Delta$ to $12.56 \Delta$ in BI prism reading glass group and from $10.05 \Delta$ to $12.71 \Delta$ in placebo reading glass group [10].

There are very few studies which uses Brock string for therapy. One such study was done by Schieman where NPC break in vision therapy/orthoptics group improved from $13.7 \mathrm{~cm}$ to $4.5 \mathrm{~cm}$ and PFV at near was $12.5 \Delta$ before therapy which improved to $31.8 \Delta$ after 12 weeks of therapy. The vision therapy group uses brock string, loose prisms, etc. during therapy [4]. In a study done in 4 CI subjects, the improvement was seen in NPC, near PFV and decreased exophoria after VT. Both home based and office based therapy was done 3 days/week for 6 weeks. Before the vergence therapy, the CI subjects were $9 \Delta \pm 1.4 \Delta$ exophoric which decreased to $3.3 \Delta \pm 1.9 \Delta$ exophoric after the vergence therapy. There was improvement in NPC break decreasing from $13.4 \mathrm{~cm} \pm 5.6 \mathrm{~cm}$ to $8.1 \mathrm{~cm} \pm 2.3 \mathrm{~cm}$. PFV at near in the same group increased from $14 \Delta \pm 4.5 \Delta$ to $40 \Delta \pm 4.1 \Delta$. For home based therapy, the patients used brock string and loose prism stick [11]. In a research done by Layali Ibrahim Hassan, 66 students underwent brock string therapy out of total 273 students. There was significant improvement in NPC from $30.9 \mathrm{~cm}$ to $19.29 \mathrm{~cm}$ and PFV from $7.67 \Delta$ to $13.81 \Delta$ [12]. Likewise, in our study the break point for NPC improved from $7.75 \mathrm{~cm}$ to $6 \mathrm{~cm}$ in case of accommodative target and in case of non-accommodative target, it improved from $10.5 \mathrm{~cm}$ to $8 \mathrm{~cm}$. At near, PFV break increased from $14 \Delta$ to $30 \Delta$. These results suggest that brock string therapy performs best in patient with convergence insufficiency. 
Kyung Min Kim reported that the exophoria before therapy was $12 \Delta$ which decreased to $4 \Delta$ after 12 weeks HBPP therapy. In this study, PFV at near improved from $11.3 \Delta$ to $19.1 \Delta$ [13]. Pencil push up test was used for therapy. In contrast, it had been reported that failure of PPT is due to lack of compliance, it is not effective and several other causes. For the failure of PPT, 87\% ophthalmologist claimed lack of compliance as the major reason and $10 \%$ claimed it as no more effective treatment [14]. Birnbaum MH concluded that vision therapy is successful in $61.9 \%$ patient who received both OBVT and HBVT and in $30 \%$ patient who received HBVT. It has also been concluded that VT is effective in eliminating asthenopia and improving convergence functions [15].

In the present study, there was significant improvement in NPC and PFV in the study and it clearly showed that the brock string therapy is efficacious in clinical significant improvement on binocular functions. It can be assumed that initially there is decreased exophoria, improvement in NPC and other vergence tests in both regular and occasional therapy group. But, the results are found to be more consistent and stable in case of regular therapy group.

In treating exophoria which is a latent strabismus, the peripheral vision is needed to process spatial information to help the person point both eyes at same place. If the central or peripheral parallel pathways are not being used effectively, it can be a huge barrier to success in treating the patient.

So, when we emphasize the peripheral visual processing of spatial information through brock string therapy, these skills are in place, then it is a matter of practicing using both eyes together at different distances and doing different activities. Ultimately, this will allow for comfortable and efficient use of both eyes at the same time.

With practice, the brain can usually learn how to pay more attention to both eyes when they are open. When this occurs, it becomes much easier for the patient to start using both eyes together. To conclude, it is better to train the brain regularly to achieve this. So, it can be assumed that regular practice of brock string therapy would give a consistent binocular vision stability than the occasional practice.

\section{Conclusion}

This study is an important first step in understanding how the vision therapy described within this research evoked clinical and functional changes in the CI subjects studied. It can be observed that NPC, NRA, PFV at near, vergence facility, accommodative facility and near exophoria improved in both regular and occasional therapy group but, the consistency of the result was seen in regular therapy group.

In conclusion, this study demonstrates that brock string therapy is effective in improving NPC and vergence disorders. This investigation is the first to assess the efficacy of brock string in regular and occasional group. The near exophoric CI subjects became significantly less and good improvement was seen in NPC and vergence tests after brock string therapy compared to each subject's baseline measurement. We can't deny the fact that both regular and occasional therapies are efficacious for the treatment of $\mathrm{CI}$ but the regular therapy shows more stable result than occasional one. So, for the smooth and proficient performance with the elimination of suppression, regular therapy is essential. After the development of essential visual skill for fixating target in brock string therapy, patient can continue therapy sitting comfortably at home.

\section{Limitations and Future Directions}

The limitation must be considered when interpreting the results of this study. The sample size of 30 patients was small, which affects the precision of our treatment effects. Also, there was lack of symptom level measurement using the questionnaire. As the therapy requires constant fixation and attention of the patient, it should be performed in the office under the supervision of vision therapist or behavioral optometrist. Patient may find it cumbersome to visit the office daily changing their regular schedule.

The age group for our study was $21.8 \pm 3.2$. A future study particularly of this age group and large sample would give a generalized result on this.

\section{Bibliography}

1. Rahul Bhola. "Binocular vision”. eyerounds.org (2006).

2. Cooper J., et al. "Convergence insufficiency-a major review". Optometry (St. Louis, Mo.) 83.4 (2012): 137-158.

3. Judith B Lavrich. "Convergence insufficiency and its current treatment". Current Opinion in Ophthalmology 21.5 (2010): 356-360. 
4. Scheiman M., et al. "A randomized clinical trial of treatments for convergence insufficiency in children". Archives of Ophthalmology 123.1 (2005): 14-24.

5. St. Louis. "Definition of optometric vision therapy". American Optometric Association (2009).

6. Debora Lee Chen. "Treatment of convergence insufficiency".

7. Tara L Alvarez. "A pilot study of disparity vergence and near dissociated phoria in convergence insufficiency patients before vs. after vergence therapy". Frontiers in Human Neuroscience (2015).

8. Aziz S., et al. "Are orthoptics Exercises an effective treatment for convergence and fusion deficiencies?". Strabismus 14.4 (2009): 183-189.

9. Mitchell Scheiman., et al. "A Randomized Clinical Trial of Vision Therapy/ Orthoptics versus Pencil Pushups for the Treatment of Convergence Insufficiency in Young Adults". Optometry and Vision Science 82.7 (2005): 583-595.

10. Scheiman M., et al. "Randomised clinical trial of the effectiveness of base-in prism reading glasses versus placebo reading glasses for symptomatic convergence insufficiency in children". The British Journal of Ophthalmology 89.10 (2005): 1318-1323.

11. Tara L Alvarez., et al. "Vision Therapy in Adults with Convergence Insufficiency: Clinical and Functional Magnetic Resonance Imaging Measures". Optometry and Vision Science 87.12 (2010): E985-1002.

12. Layali Ibrahim Hassan., et al. "Efficacy of home-based vision therapy for convergence insufficiency in secondary schools' students". Sudanese Journal of Ophthalmology 9.1 (2017): 5-9.

13. Kyung Min Kim., et al. "Effectiveness of home-based pencil push-ups (HBPP) for patients with symptomatic convergence insufficiency". Korean Journal of Ophthalmology 25.3 (2011): 185-188.

14. Sourabh Patwardhan., et al. "Preferred clinical practice in convergence insufficiency in India: A survey". Indian Journal of Ophthalmology 56.4 (2008): 303-306.
15. Birnbaum MH., et al. "Efficacy of vision therapy for convergence insufficiency in an adult male population". Journal of American Optometric Association 70.4 (1999): 225-232.

\section{Assets from publication with us}

- Prompt Acknowledgement after receiving the article

- Thorough Double blinded peer review

- Rapid Publication

- Issue of Publication Certificate

- High visibility of your Published work

Website: www.actascientific.com/

Submit Article: www.actascientific.com/submission.php

Email us: editor@actascientific.com

Contact us: +919182824667 